\title{
An Objective Climatology of Tropical Cyclone Diurnal Pulses in the Atlantic Basin
}

\author{
Sarah D. Ditchek, John Molinari, Kristen L. Corbosiero, And Robert G. Fovell \\ Department of Atmospheric and Environmental Sciences, University at Albany, State University of New York, \\ Albany, New York
}

(Manuscript received 18 October 2018, in final form 2 December 2018)

\begin{abstract}
Storm-centered IR brightness temperature imagery was used to create 6-h IR brightness temperature difference fields for all Atlantic basin tropical cyclones from 1982 to 2017. Pulses of colder cloud tops were defined objectively by determining critical thresholds for the magnitude of the IR differences, areal coverage of cold-cloud tops, and longevity. Long-lived cooling pulses ( $\geq 9 \mathrm{~h})$ were present on $45 \%$ of days overall, occurring on $80 \%$ of major hurricane days, $64 \%$ of minor hurricane days, $46 \%$ of tropical storm days, and $24 \%$ of tropical depression days. These cooling pulses propagated outward between 8 and $14 \mathrm{~m} \mathrm{~s}^{-1}$. Short-lived cooling pulses $(3-6 \mathrm{~h})$ were found $26.4 \%$ of the time. Some days without cooling pulses had events of the opposite sign, which were labeled warming pulses. Long-lived warming pulses occurred $8.5 \%$ of the time and propagated outward at the same speed as their cooling pulse counterparts. Only $12.2 \%$ of days had no pulses that met the criteria, indicating that pulsing is nearly ubiquitous in tropical cyclones. The environment prior to outward propagation of cooling pulses differed from warming pulse and no pulse days by having more favorable conditions between 0000 and $0300 \mathrm{LT}$ for enhanced inner-core convection: higher SST and ocean heat content, more moisture throughout the troposphere, and stronger low-level vorticity and upper-level divergence.
\end{abstract}

\section{Introduction}

The tropical cyclone (TC) diurnal cycle has been studied using satellite infrared (IR) data (Browner et al. 1977; Muramatsu 1983; Steranka et al. 1984; Lajoie and Butterworth 1984; Kossin 2002; Dunion et al. 2014; Wu and Ruan 2016), the Tropical Rainfall Measuring Mission (TRMM; Jiang et al. 2011; Bowman and Fowler 2015; Wu et al. 2015; Leppert and Cecil 2016), lightning data (Stevenson et al. 2016), rawinsondes and dropsondes (Frank 1977; Duran and Molinari 2016), numerical modeling (Hobgood 1986; Ge et al. 2014; Melhauser and Zhang 2014; Navarro and Hakim 2016; Navarro et al. 2017), and a high-resolution CPC morphing technique dataset (HCMORPH; Shu et al. 2013).

A schematic representing the studies in the literature that used observations to study the TC diurnal cycle is given in Fig. 1. It reveals a consensus on the timing of the TC diurnal cycle. Overnight, lightning (red shading) peaks in the inner $300 \mathrm{~km}$ of the TC at around 0000 local time (LT). This enhanced lightning activity occurs during a period of enhanced convection, which reaches its maximum

Corresponding author: Sarah D. Ditchek, sarahditchek@gmail.com in the early morning hours, producing the coldest cloud tops at around 0400 LT (orange shading). Approximately two hours later, precipitation peaks in the inner core (yellow shading). Beginning at around 0600 LT, the cirrus canopy expands radially outward and reaches its maximum areal extent around 1800 LT (green shading).

The observational studies in Fig. 1 on the diurnal cycle of the cirrus canopy focused on its areal expansion. Taking a different approach, Dunion et al. (2014) used 3-h GOES 4-km IR brightness temperature imagery to look at 6-h IR brightness temperature differences of North Atlantic major hurricanes from 2001 to 2010. They found that an area of colder cloud tops propagated outward at around $5-10 \mathrm{~m} \mathrm{~s}^{-1}$ over the course of the day, with warming on its inside edge. This "diurnal pulse," which they showed might be coupled to convection, began between 0400 and $0800 \mathrm{LT}$ at $r=200 \mathrm{~km}$ and ended between 1500 and $1800 \mathrm{LT}$ at $r=500 \mathrm{~km}$. Their findings suggest that diurnal pulses might be a prominent feature of the TC diurnal cycle.

Here, the results of Dunion et al. (2014) were expanded to include all Atlantic basin TCs over the 36-yr period of 1982-2017. Storm-centered GridSat-B1 IR brightness temperature imagery (Knapp et al. 2011) were used to create 6-h IR brightness temperature difference fields as 


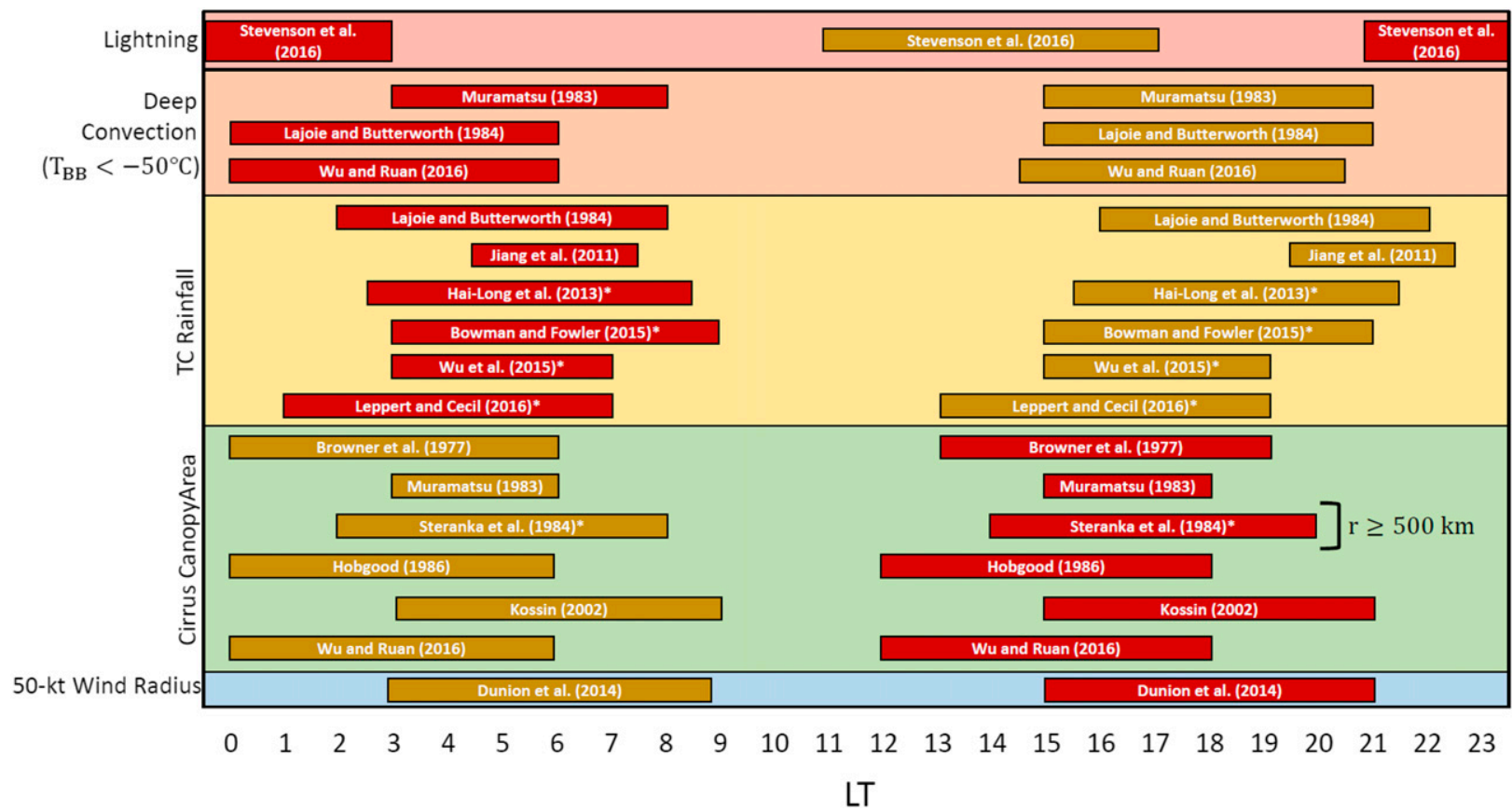

FIG. 1. A graphical view of results from papers on the observed TC diurnal cycle. Papers are indicated by the author(s) and year of publication. The papers' findings regarding the timing of the TC diurnal cycle are organized by local time (LT) on the $x$ axis. The maroon and gold boxes indicate the range of time when a variable's diurnal cycle is maximized or minimized, respectively. Variables are denoted along the $y$ axis and each variable is associated with a different color block. An asterisk within a box indicates that a formal diurnal harmonic calculation was conducted in that study. In those cases, the width of the maroon and gold boxes corresponds to $\pm 3 \mathrm{~h}$ of the maximum and minimum value of the diurnal harmonic for the given variable. If no asterisk is present, then the width of the boxes corresponds to the range of time associated with the diurnal cycle found in the text and figures of each paper.

in Dunion et al. (2014) (section 2). To identify diurnal pulses in the difference fields with similar phasing as Dunion et al. (2014), an objective metric was created (section 3). This metric identified two different types of diurnal pulses: cooling pulses and warming pulses. Cooling pulses are similar to those documented in Dunion et al. (2014) and are an area of cooler cloud tops that propagates outward over the course of the day. Warming pulses are a previously undocumented pulse type of warmer cloud tops that propagate outward over the course of the day with the same timing and speed as the Dunion et al. (2014) pulse. The 6-h IR brightness temperature evolution and environmental differences for days where a diurnal pulse does and does not exist were then compared and contrasted (section 4). Additionally, the following questions were answered (section 4) for both the cooling and warming pulse types identified by the objective metric:

- What is the propagation speed of diurnal pulses?

- How frequently do diurnal pulses occur in the Atlantic basin?

- Do diurnal pulses occur more frequently in storms of higher intensity?

- Is the frequency and orientation of the diurnal pulse influenced by vertical wind shear?

- How axisymmetric are diurnal pulses?
A succinct comparison between cooling and warming pulses, including answers to the above questions, is found in section 5 .

\section{Data}

Four times daily, Atlantic-basin TC location and intensity over the 36-yr period of 1982-2017 were obtained from the NHC "best track" hurricane database (HURDAT2; Landsea and Franklin 2013). For each storm, the HURDAT2 data were linearly interpolated to a temporal resolution of $3 \mathrm{~h}$. These years were chosen since the Statistical Hurricane Intensity Prediction Scheme (SHIPS; DeMaria and Kaplan 1994, 1999; DeMaria et al. 2005) database for storms in the Atlantic basin covers the same period. Cases that satisfied the following criteria were studied: 1) storms of tropical depression or greater strength, 2) storms that were not extratropical or subtropical, 3) storm times with storm centers south of $40^{\circ} \mathrm{N}$, and 4) storm times before a storm made landfall for greater than $6 \mathrm{~h}$.

For each storm time, the $p=850-200 \mathrm{hPa}, r=0-500-\mathrm{km}$ SHIPS shear magnitude (kt) and direction $\left({ }^{\circ}\right)$ were retrieved, linearly interpolated to 3-h temporal resolution, and converted to meters per second $\left(\mathrm{m} \mathrm{s}^{-1}\right)$. 

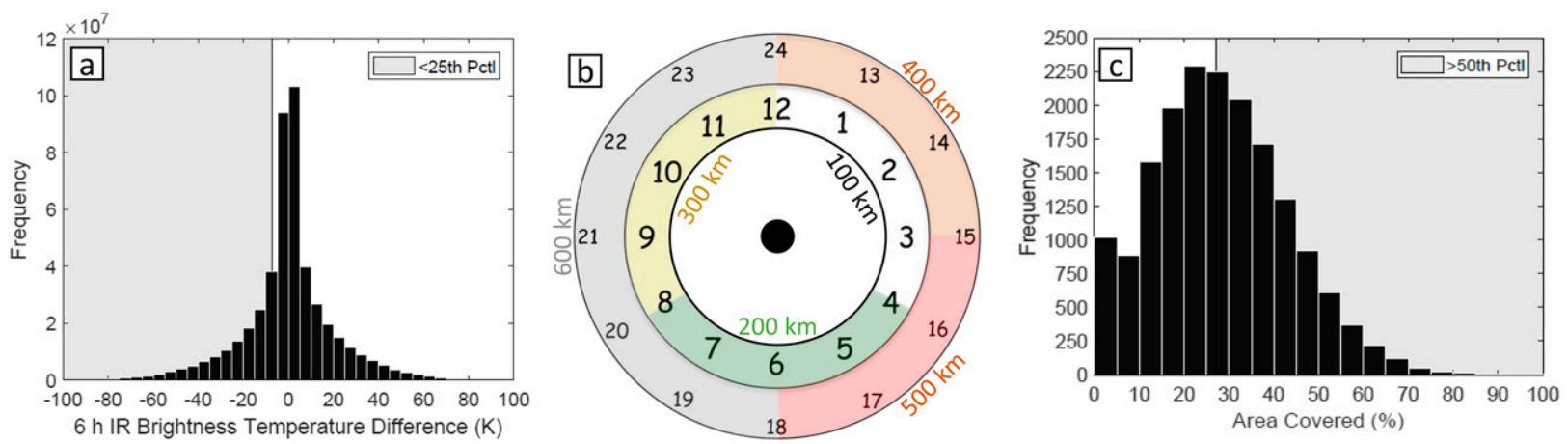

FIG. 2. Three of the four steps to identify days when diurnal cooling pulses occurred. (a) Histogram (black shading) of all 6-h IR brightness temperature differences $(K)$. The lower quartile $(<25$ th percentile, gray shading) represents the threshold chosen to identify cooling cloud tops. (b) An extended version of the Dunion et al. (2014) diurnal clock schematic. The clock depicts the approximate radius (colored text) where the diurnal pulse should be in each LT window (shaded). The clock was extended to include the 0000-0400 LT window (white shading), corresponding to the 100-km radius, and the 1800-0000 LT window (gray shading), corresponding to the 600-km radius. This clock was used to create the objective diurnal pulse identification procedure. (c) Histogram (black shading) of the area covered (\%) by the cooling cloud tops identified in (a). The upper half ( $>50$ th percentile, gray shading) represents the threshold chosen to identify a day when a diurnal pulse occurred. A similar procedure was used to identify days when diurnal warming pulses occurred.

From this distribution, low-shear (lower quartile, $<4.4 \mathrm{~m} \mathrm{~s}^{-1}$ ), moderate-shear (interquartile range, $4.4-$ $10.6 \mathrm{~m} \mathrm{~s}^{-1}$, and high-shear (upper quartile, $>10.6 \mathrm{~m} \mathrm{~s}^{-1}$ ) groupings were defined (not shown).

Environmental fields were also retrieved from SHIPS for each storm time, including daily SST; ocean heat content (OHC); low-, mid-, and upper- level relative humidity (RH); 200-hPa divergence; $850-\mathrm{hPa}$ vorticity; total precipitable water (TPW); and $1000-\mathrm{hPa}$ equivalent potential temperature $\theta_{e}$. These data come from the National Centers for Environmental Prediction (NCEP) global model analyses, Global Forecast System (GFS) analysis, and satellite altimetry data.

The GridSat-B1 IR brightness temperature dataset has a temporal resolution of $3 \mathrm{~h}$ and a horizontal resolution of $0.07^{\circ} \times 0.07^{\circ}$ on a latitude equal-angle grid (Knapp et al. 2011). For each storm time, IR brightness temperature was extracted centered on the storm, yielding 17415 storm-relative grids over 2613 storm days that encompassed 466 individual storms. Then, for each storm, 6-h IR brightness temperature differences were computed on the $0.07^{\circ} \times 0.07^{\circ}$ resolution grid every $3 \mathrm{~h}$.

\section{Objective diurnal pulse identification}

In the 6-h IR brightness temperature difference fields of Dunion et al. (2014), the diurnal pulse was identified as an area of cooling cloud tops (seen as negative values of brightness temperature differences) that propagated away from the storm center over the course of the day. They created a schematic that identified at which radius the diurnal pulse should exist based on the time of day. An extended version of their schematic is given in Fig. 2b.
It is of interest to determine how general and common the Dunion et al. (2014) cooling pulse is in the more extensive dataset presented here. First, data from all days were sorted into bins centered on the hour and averaged. An outward-propagating signal that matched the Dunion et al. (2014) pulse was found to be the dominant signal in the unfiltered data (see appendix A). A four-step procedure was then created to objectively identify and extract diurnal pulses from this unfiltered data. Data were initially divided into two groups: days that had cooling pulses similar to those found in Dunion et al. (2014) and days that did not. The cooling pulses were further divided into long lived and short lived based on their longevity. Subsequently, it was discovered that some days that did not have cooling pulses had outward-propagating warming pulses instead. Therefore, days that did not have cooling pulses were classified into long-lived and short-lived warming pulses and into days that had no conforming outward-propagating signals.

\section{a. Identification of cooling pulses}

First, cooling cloud tops in each 6-h IR brightness temperature difference field were identified. In this study, cooling cloud tops were defined as the lower quartile (Fig. $2 \mathrm{a},<-7.1 \mathrm{~K}$, gray shading) of the 6-h IR brightness temperature difference values for all storm times. Second, the spatial location of the cooling cloud tops had to follow the diurnal clock schematic (Fig. 2b). The LT window where each storm time fell was determined. Then, cooling cloud tops were sought within $\pm 100 \mathrm{~km}$ of the radius associated with each LT window. For example, a storm time of 0700 LT falls in the 0400 0800 LT window, so cooling cloud tops were identified 
between $r=100$ and $300 \mathrm{~km}$. Third, for storm times with cooling cloud tops that followed the diurnal clock schematic, the percent of a radial bin covered by the cooling cloud tops was calculated. The storm times that fell in the upper half of that areal distribution (Fig. 2c, $>27 \%$, gray shading) were identified. Last, the number of hours in a given day where cooling cloud tops that followed the diurnal clock schematic and satisfied the areal criterion was calculated (Fig. 3). This accounts for outward propagation, since over each storm day the objective metric cycles from the $0-200-\mathrm{km}$ radius for the LT window of $0000-0400 \mathrm{LT}$ to the $500-700-\mathrm{km}$ radius for the LT window of 1800-0000 LT.

On a given day, if cooling cloud tops that satisfied the areal criterion existed for $\geq 9 \mathrm{~h}$, that day was categorized as having a long-lived cooling pulse (LLCP; 1178 total days; Fig. 3, red bars in dark gray shading). If cooling cloud tops on a given day that satisfied the areal criterion existed for $t=3-6 \mathrm{~h}$, that day was categorized as a day having a short-lived cooling pulse (SLCP; 690 total days; Fig. 3, red bars in light gray shading). Using these criteria, 1868 of 2613 days $(71.5 \%)$ had a cooling pulse of some minimum duration.

\section{b. Identification of warming pulses and no-pulse days}

Days that did not have cooling cloud tops, had cooling cloud tops that did not satisfy the areal criterion, or had cooling cloud tops that satisfied the areal criterion but existed for less than $3 \mathrm{~h}$, were characterized as days that did not have diurnal cooling pulses. Further analysis revealed that some of these 745 days did have outwardpropagating features that were the opposite sign of the Dunion et al. (2014) pulses. Therefore, the four-step procedure listed above was repeated for this subset of days with the modification that the first step identified warming cloud tops, defined as the upper quartile ( $>6.6 \mathrm{~K}$, not shown) of the 6-h IR brightness temperature difference values for the subset ${ }^{1}$ (Fig. 2a). Categorizations mirrored those for the cooling pulses above: there were 222 long-lived warming pulses (LLWPs; Fig. 3, blue bars in dark gray shading) and 203 short-lived warming pulses (SLWPs; Fig. 3, blue bars in dark gray shading).

This left only 320 days ( $12.2 \%$ of the original total) that had no identifiable outward-propagating cooling or warming signal, had cooling or warming cloud tops that did not satisfy the areal criterion, or had cooling or warming cloud tops that satisfied the areal criterion but existed for $<3 \mathrm{~h}$ (NP; Fig. 3, black bars in white shading).

\footnotetext{
${ }^{1}$ Using the upper quartile of the 6-h IR brightness temperature difference values for all storm times yield qualitatively similar results.
}

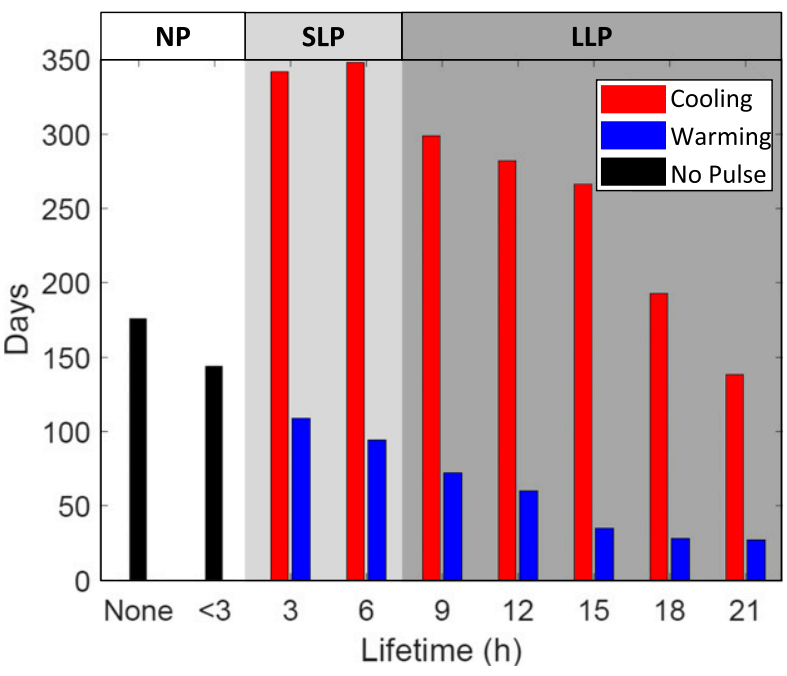

FIG. 3. The fourth step to identify days when diurnal pulses occurred. Bar chart of hours in a given day when a diurnal pulse was identified. Diurnal pulses that exist for $\geq 9 \mathrm{~h}$ on a given day were categorized as long-lived pulses (LLPs, dark gray shading). Those that exist for 3-6h on a given day were categorized as short-lived pulses (SLPs, light gray shading). All other days were categorized as days that had no pulses (NPs, white shading). The red bars represent the cooling pulses, pulses identified in the 6-h IR brightness temperature difference fields by warm colors, similar to those found in Dunion et al. (2014). The blue bars represent the warming pulses, pulses identified in the 6-h IR brightness temperature difference fields by cool colors. Black bars represent days that had no pulses.

\section{c. Summary}

Over all storm days, long-lived pulses occurred 53.6\% of the time, with LLCPs occurring $45.1 \%$ of the time and LLWPs occurring $8.5 \%$ of the time. Short-lived pulses occurred $34.2 \%$ of the time, with SLCPs occurring $26.4 \%$ of the time and SLWPs occurring $7.8 \%$ of the time. NPs occurred $12.2 \%$ of the time. Thus, pulsing behavior, whether cooling or warming and whether long lived or short lived, was by far the most common resultoccurring $87.8 \%$ of the time.

Hereafter, only LLCPs, LLWPs, and NPs will be discussed, as long-lived pulses persisted over a longer period of the day and are the more frequent diurnal pulse types compared to short-lived pulses. ${ }^{2}$

\section{Results}

\section{a. Structural evolution}

The 6-h IR composite brightness temperature evolutions for LLCP, LLWP, and NP days every $3 \mathrm{~h}$ from 0000 to 2100 LT are given in Figs. 4-6 in Earth-relative

\footnotetext{
${ }^{2}$ For a discussion of SLCPs, see appendix B.
} 
coordinates. Overlaid on each plan-view plot are black range rings every $100 \mathrm{~km}$. The white range rings correspond to the expected timing of the observed TC diurnal pulse as documented by the extended version of the Dunion et al. (2014) diurnal clock (Fig. 2b). Similar planview results were found for the spatial evolution of 6-h IR brightness temperature differences for days with similar intensities and similar shear values (not shown).

For LLCPs, as early as 0000 LT, decreasing cloud-top temperatures (green to red shading) were present in the inner core, indicative of enhanced, overnight deep convection. This trend intensified and peaked around $0000 \mathrm{LT}$, consistent with the timing of the diurnal cycle of deep convection found in the literature (Fig. 1, orange shading). By $0600 \mathrm{LT}$, the diurnal pulse had begun propagating outward and was present at $r=200 \mathrm{~km}$. The pulse continued to move outward until $1500 \mathrm{LT}$ when it reached $r=$ $500 \mathrm{~km}$. By $1800 \mathrm{LT}$, the diurnal pulse was diffuse and was located beyond $r=600 \mathrm{~km}$. This composite shows a robust cooling pulse that is by definition consistent with the timing found in the Dunion et al. (2014) clock. Additionally, as in Dunion et al. (2014), there is a warming signature (blue shading) on the inner edge of the diurnal pulse. Reproducing Fig. 4 in shear-relative coordinates (not shown) revealed that the structural asymmetries present (i.e., the strongest cooling east and northeast of the center) were due to predominantly southwesterly vertical wind shear.

In comparison, Fig. 5 depicts the 6-h IR brightness temperature difference evolution on days where LLWPs occurred. Unlike LLCP days, cloud-top temperature increased over time beginning at $0300 \mathrm{LT}$. These warmer cloud tops moved outward between 0600 and 1200 LT at a similar speed as the composite LLCP in Fig. 4. Apart from being opposite in sign, LLWPs closely resembled their cooling counterparts, including being trailed by the opposite sign temperature perturbation. As for LLCPs, the structural asymmetries seen in Fig. 5 were due to vertical wind shear (not shown).

Figure 6 depicts 6-h IR brightness temperature difference evolution on days where no outward propagation was found. Unlike in Figs. 4-5, no coherent pattern was found.

\section{b. Axisymmetric framework}

Azimuthal averages of the 6-h IR brightness temperature difference fields on days where LLCPs, LLWPs, and NPs occurred were computed using a $0.07^{\circ}$ bin size, matching the GridSat-B1 resolution, and were sorted into bins centered on the hour, and were averaged. Taking azimuthal averages allows for the calculation of the propagation speed of the diurnal pulse seen in Figs. 4-6.

Fourier harmonic analysis was then applied at each radius by fitting a series of sines and cosines to the data through least squares regression analysis in the following form:

$$
X_{i}(t)=X_{0}+\sum_{k=1}^{N}\left[a_{k} \cos \left(\frac{2 \pi k t}{\tau}\right)+b_{k} \sin \left(\frac{2 \pi k t}{\tau}\right)\right]+\varepsilon_{i}
$$

where $i$ is the bin number; $X_{i}$ is the variable in question; $t$ is the LT; $X_{0}$ is the mean value of the variable; $k$ is the harmonic index; $N$ is the maximum number of harmonics fit to the data; $a_{k}$ and $b_{k}$ are the amplitudes of the cosine and sine terms of the $k$ th harmonic, respectively; $\tau$ is the length of the day; and $\varepsilon_{i}$ is the residual.

The azimuthally averaged evolution on days where LLCPs occurred is depicted in Fig. 7a. Unsurprisingly, the outward-propagating diurnal pulse found in Fig. 4 is seen in the azimuthal mean. Overnight, between 0000 and $0500 \mathrm{LT}$, enhanced convection peaks in the inner core from $r=0$ to $200 \mathrm{~km}$. Afterward, the diurnal pulse begins to propagate outward. On average, the outward-propagation speed of the diurnal pulse between $r=200$ and $350 \mathrm{~km}$ is $8.33 \mathrm{~m} \mathrm{~s}^{-1}$, falling within the propagation speed range $\left(5-10 \mathrm{~m} \mathrm{~s}^{-1}\right)$ found in Dunion et al. (2014) (black lines). The outward-propagation speed between $r=350$ and $500 \mathrm{~km}$ is $13.89 \mathrm{~m} \mathrm{~s}^{-1}$, falling outside of the propagation speed range found in Dunion et al. (2014) (black lines).

As expected from the results given in Fig. 5, the azimuthally averaged evolution on days where LLWPs occurred also has an outward-propagating signal (Fig. 7c). In many respects, this feature is the inverse of the cooling pulse, where a warming of cloud tops moves outward. Since this warming had a similar propagation speed as the cooling pulse found in Figs. 7a,b, they probably propagate outward by the same mechanism. Dunion et al. (2014) and recent research has suggested that cooling pulses act like internal inertial gravity waves (Navarro et al. 2017; O'Neill et al. 2017). Intensification, and collapse, of convective activity and heating can each generate gravity waves of opposite signs. Therefore, the identification that warming pulses are typically present when cooling pulses are not provides evidence for a gravity wave interpretation of diurnal pulses.

While warming and cooling pulses had similar propagation speeds, the structure of the diurnal harmonic differed. In the inner $200 \mathrm{~km}$, LLCPs had cooling cloud tops from 0000 to $0600 \mathrm{LT}$ (Fig. 7b). Later in the day, there was warming radially inward of the outward-propagating cooling cloud tops. The diurnal harmonic was more pronounced between $r=200$ and $500 \mathrm{~km}$, the radii where the Dunion et al. (2014) pulse is prominent. On the other hand, the diurnal harmonic for LLWPs was maximized at inner radii (Fig. 7d). Warming cloud tops occurred at inner radii between 0000 and $1200 \mathrm{LT}$ and were followed by a period of cooling cloud tops. While LLWPs do propagate outward, they do not have as strong a diurnal harmonic as the LLCPs beyond $r=200 \mathrm{~km}$. 


\section{LLCP}
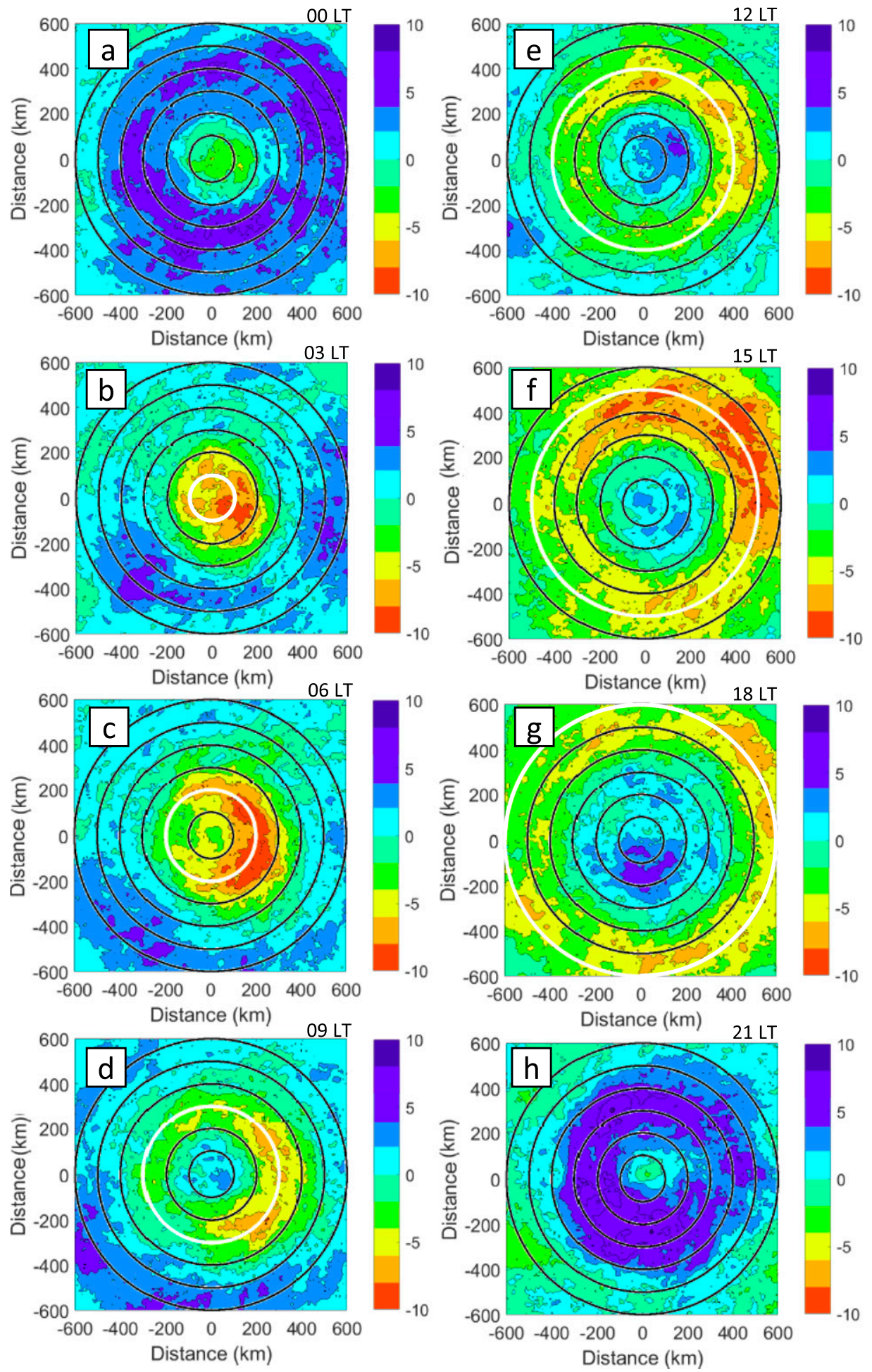

FIG. 4. A plan view of composite 6-h IR brightness temperature difference fields (K) ending at (a) 0000, (b) 0300, (c) 0600, (d) 0900, (e) 1200, (f) 1500, (g) 1800, and (h) 2100 LT for days when LLCPs occurred. The contour interval is every $2 \mathrm{~K}$. Black range rings are overlaid on each image every $100 \mathrm{~km}$ from $r=100$ to $600 \mathrm{~km}$. White range rings indicate the time at which the pulse should occur using the extended version of the Dunion et al. (2014) diurnal clock (Fig. 2b). 


\section{LLWP}
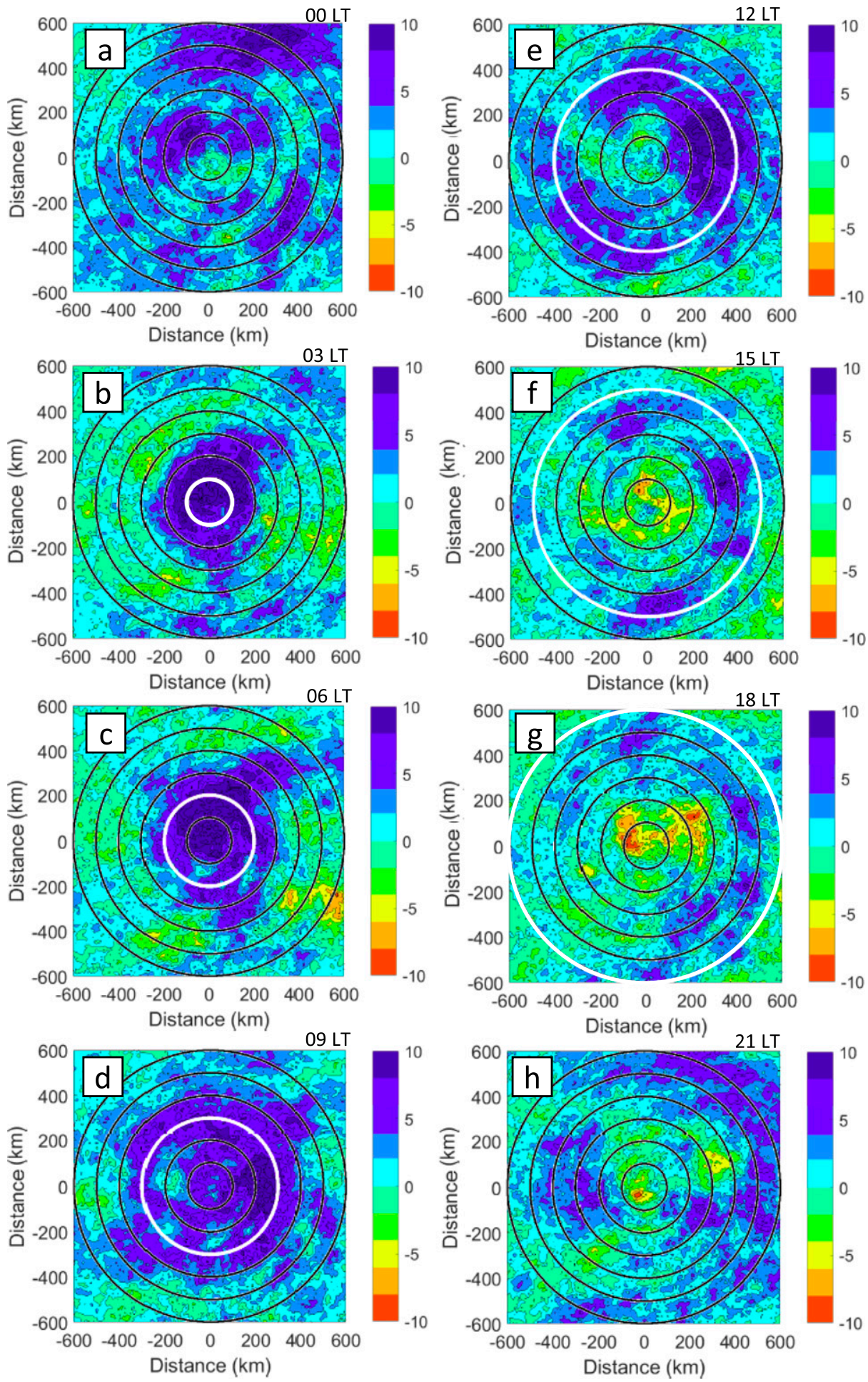

FIG. 5. As in Fig. 4, but for days when LLWPs occurred. 
NP
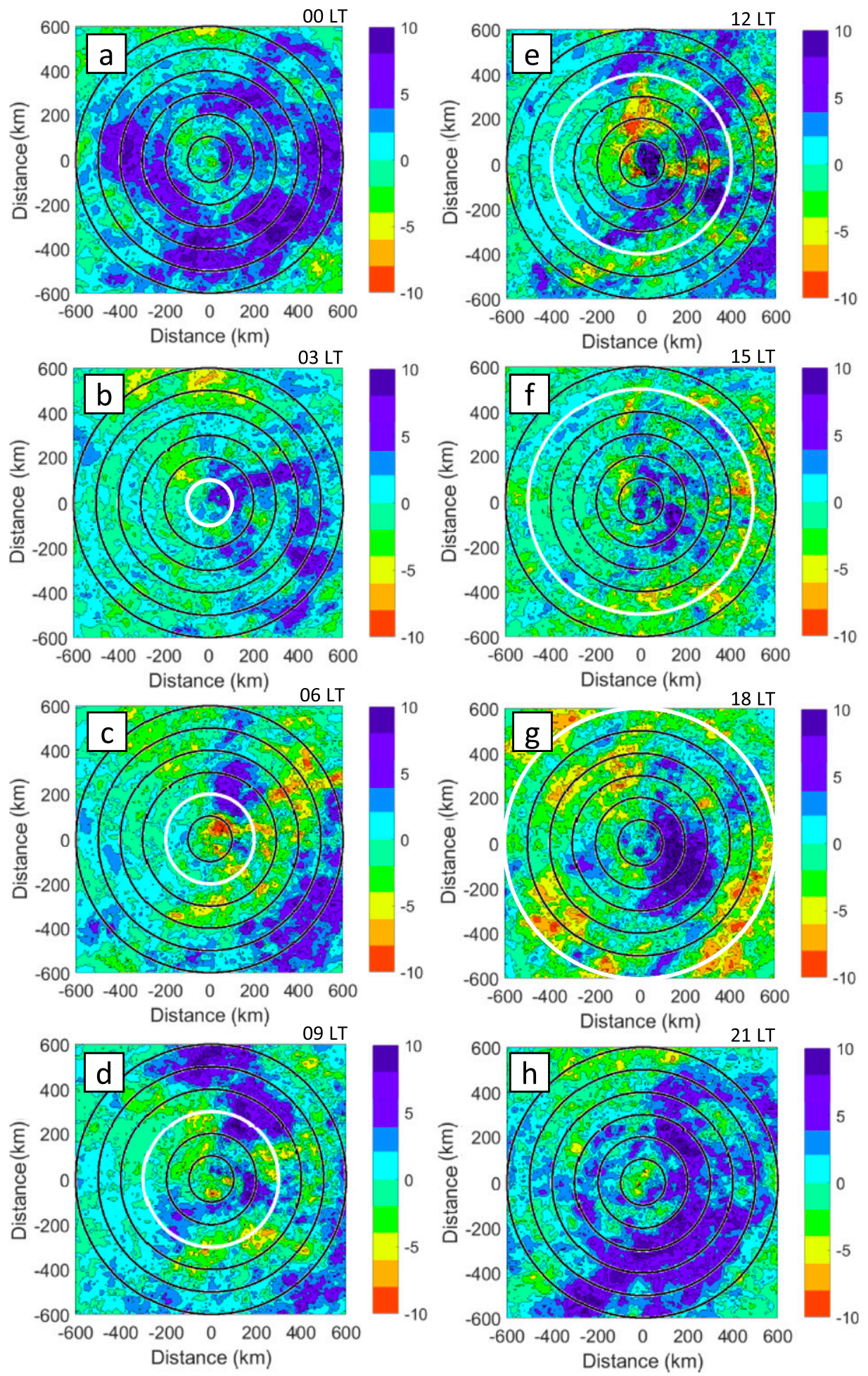

FIG. 6. As in Fig. 4, but for days when NPs occurred. 


\section{Azimuthal Average}
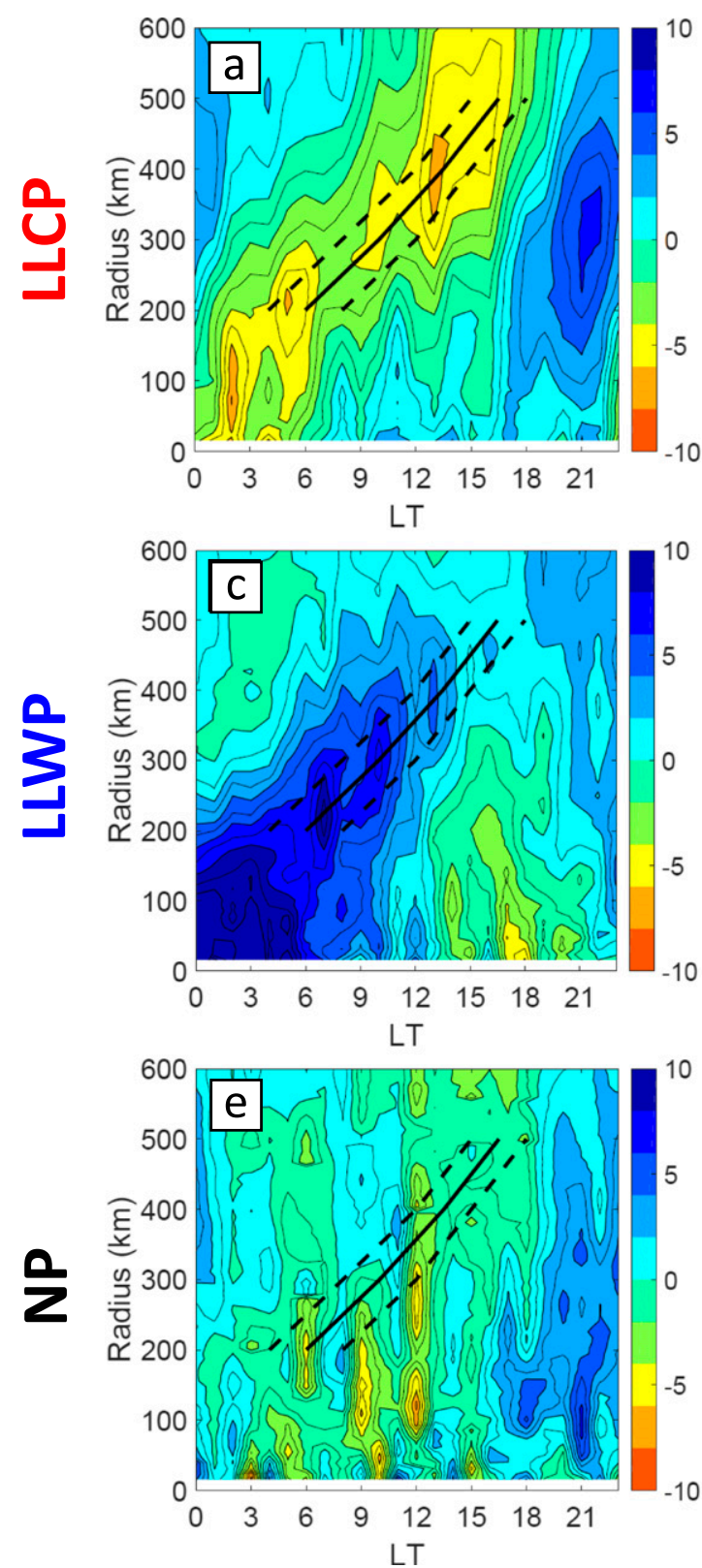

Diurnal Harmonic
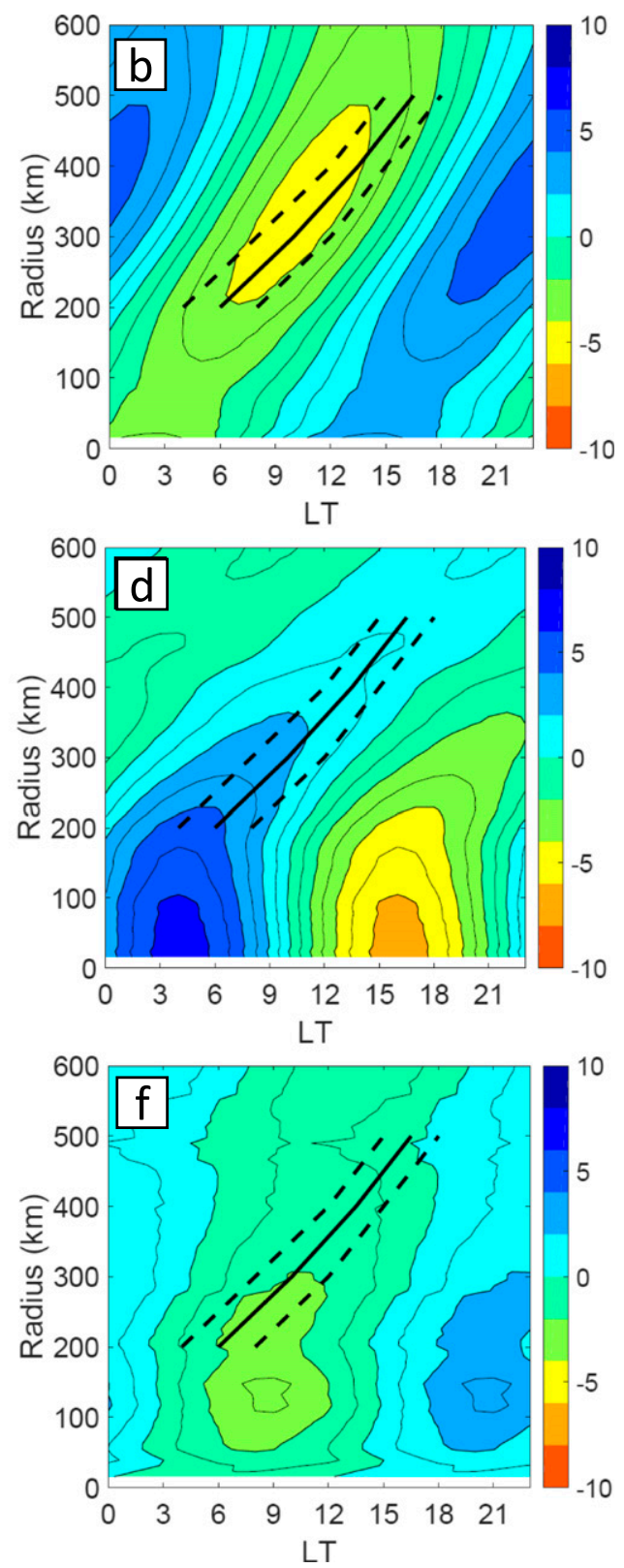

FIG. 7. (a),(c),(e) Azimuthal averages and their corresponding (b),(d),(f) diurnal harmonics for (a),(b) LLCPs; (c),(d) LLWPs; and (e),(f) NPs. Contour interval is every $2 \mathrm{~K}$. Black lines correspond to the mean (solid), earliest, and latest (dashed) times of the onset of the diurnal pulse according to the original diurnal clock in Dunion et al. (2014).

Figure 7e depicts the azimuthally averaged evolution on days when no pulse occurred. The field is noisy, and the corresponding diurnal harmonic (Fig. 7f) does not show a pulse signature.

\section{c. Frequency}

Figure 8a depicts how frequently LLCPs, LLWPs, and NPs were detected for each intensity category, where intensity was taken as the maximum intensity reached on a given day. LLCPs occurred $79.5 \%$ of the time on days where major hurricane intensity was reached. Pulse frequency decreased with decreasing intensity, with LLCPs occurring $63.8 \%, 46.0 \%$, and $23.7 \%$ of the time on days when minor hurricane, tropical storm, and tropical depression intensity was reached, respectively. 

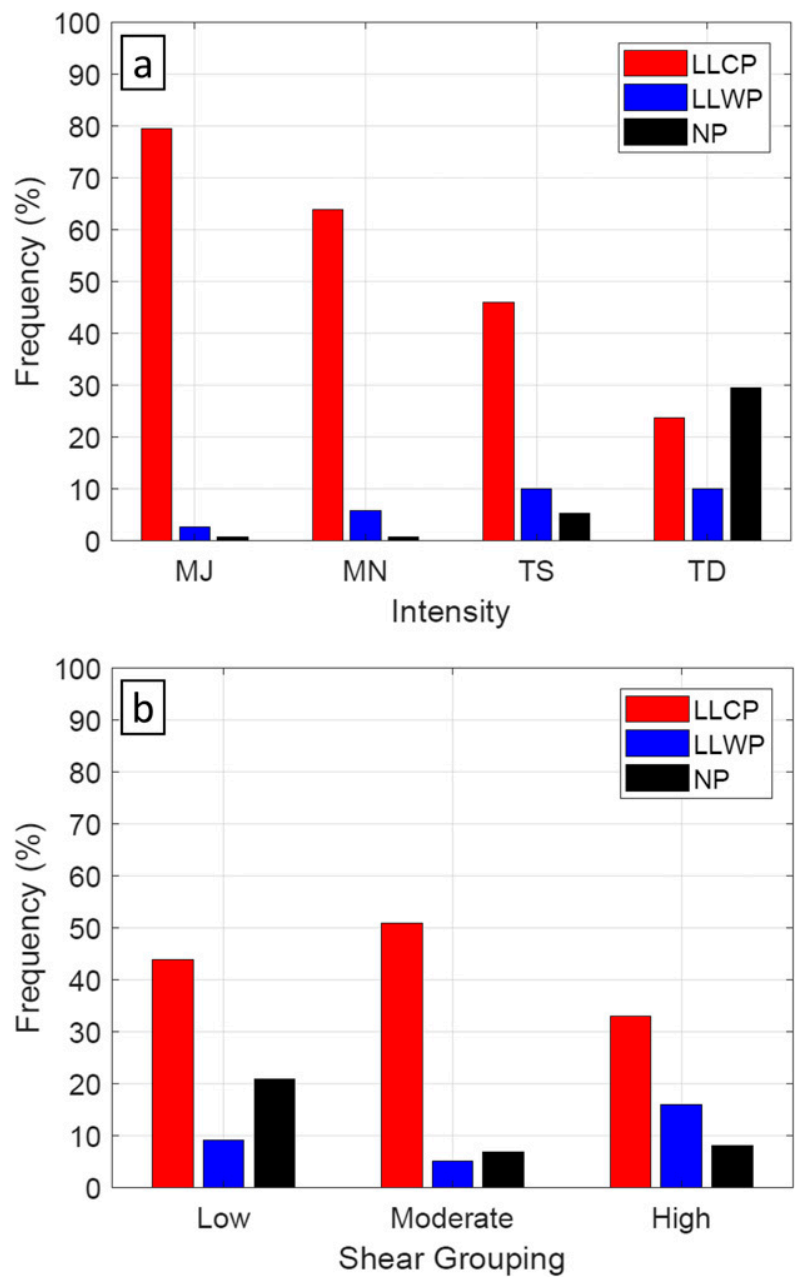

FIG. 8. Bar chart depicting the percentage of time LLCPs (red bars), LLWPs (blue bars), and NPs (black bars) occurred (a) for each intensity category [(major hurricane (MJ), minor hurricane (MN), tropical storm (TS), and tropical depression (TD)] and (b) for each shear grouping (low, moderate, and high).

LLWPs and NPs, however, occurred most frequently in tropical depressions: $10.1 \%$ and $29.5 \%$ of the time, respectively. With increasing intensity, LLWP and NP occurrence decreased, with only $2.7 \%$ and $0.8 \%$ of major hurricanes having LLWPs and NPs, respectively. Overall, NP classifications were rare in storms that were greater than tropical depression intensity, occurring only $6.9 \%$ of the time. Therefore, virtually all developed storms had pulses, dominated by cooling pulses.

Figure $8 \mathrm{~b}$ depicts the influence of vertical wind shear on pulse frequency. Shear was measured as the average magnitude on a given day. LLCPs occurred $43.8 \%$ and $50.8 \%$ of the time on days where the average shear magnitude fell in the low- and moderate- shear grouping, respectively. The percentage of LLCP occurrences decreased on high-shear days to $33.0 \%$. On the other
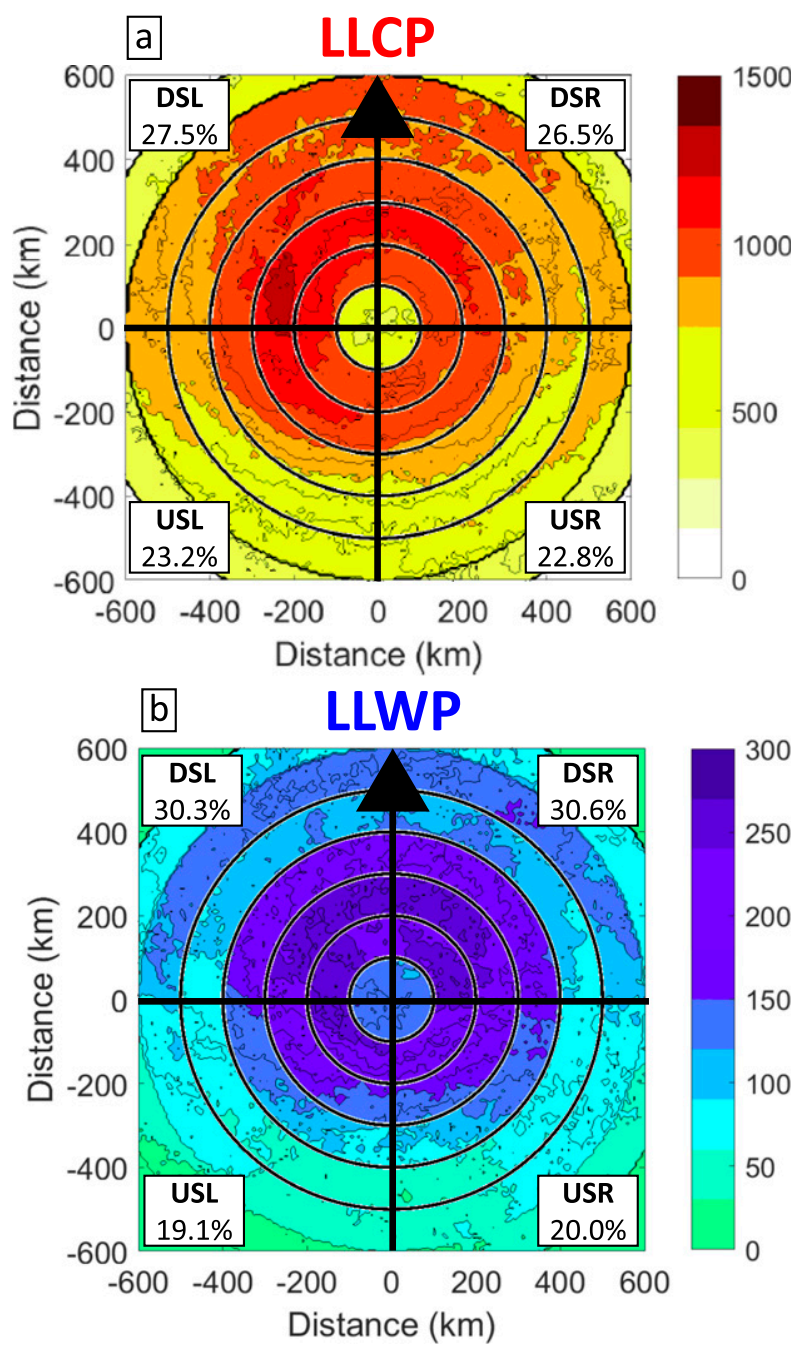

FIG. 9. A plan-view composite of the spatial location of all (a) LLCP and (b) LLWP occurrences. The shear vector points to the top of each plot (black triangle). Black range rings are overlaid on each image every $100 \mathrm{~km}$ from $r=100$ to $600 \mathrm{~km}$. Black lines divide the figures into downshear-left (DSL), downshear-right (DSR), upshear-right (USR), and upshear-left (USL) quadrants. Percentages indicate the frequency of pulse occurrence in each quadrant. Note the different scale since LLWPs occurred less frequently than LLCPs.

hand, LLWPs occurred most often in high-shear environments: $15.9 \%$ of the time compared to $9.0 \%$ of the time in low-shear environments. NPs occurred $20.9 \%$ of the time in low-shear environments. Their frequency decreased with increasing shear magnitude, with only $8.1 \%$ occurring in high-shear environments. Overall, there is less of a dependence on shear than on intensity.

\section{d. Shear-relative LLCP and LLWP location}

On days when LLCPs occurred, Fig. 9a shows their shear-relative location for all intensity and shear categories. 
TABLE 1. The mean and standard deviation of variables from 0000 to 0300 LT on LLCP, LLWP, and NP days. Significance differences were calculated at the $99 \%$ confidence level assuming both equal and then unequal variances using a Student's $t$ test. Statistically significant differences between each of the three individual groups (i.e., LLCP, LLWP, and NP) are indicated by $\dagger,{ }^{*}$, and + , respectively.

\begin{tabular}{lllccrc}
\hline \hline Variable & Units & \multicolumn{1}{c}{ Radius } & Pressure & LLCP $(\dagger)$ & LLWP $(*)$ & NP $(+)$ \\
\hline IR & $\mathrm{K}$ & $r=0-200 \mathrm{~km}$ & - & $237.3 \pm 29.5^{*,+}$ & $254.6 \pm 31.1^{\dagger}$ & $261.5 \pm 31.0^{\dagger}$ \\
Daily SST & ${ }^{\circ} \mathrm{C}$ & $r=0-50 \mathrm{~km}$ & - & $27.5 \pm 1.4^{*,+}$ & $26.6 \pm 1.6^{\dagger}$ & $26.7 \pm 1.8^{\dagger}$ \\
OHC & $\mathrm{KJ} \mathrm{cm}{ }^{-2}$ & - & - & $46.9 \pm 29.6^{*},+$ & $32.9 \pm 25.1^{\dagger}$ & $34.8 \pm 27.3^{\dagger}$ \\
$\mathrm{TPW}$ & $\mathrm{mm}$ & $r=0-500 \mathrm{~km}$ & - & $52.7 \pm 5.6^{*,+}$ & $48.8 \pm 6.2^{\dagger}$ & $47.3 \pm 6.0^{\dagger}$ \\
$\Theta_{e}$ & $\mathrm{~K}$ & $r=200-800 \mathrm{~km}$ & $p=1000 \mathrm{hPa}$ & $348.6 \pm 5.5^{*,+}$ & $345.2 \pm 6.0^{\dagger}$ & $345.1 \pm 6.4^{\dagger}$ \\
$\mathrm{RH}_{\text {lower }}$ & $\%$ & $r=200-800 \mathrm{~km}$ & $p=850-700 \mathrm{hPa}$ & $68.5 \pm 7.3^{*,+}$ & $66.1 \pm 7.3^{\dagger}$ & $65.9 \pm 7.6^{\dagger}$ \\
$\mathrm{RH}_{\text {middle }}$ & $\%$ & $r=200-800 \mathrm{~km}$ & $p=700-500 \mathrm{hPa}$ & $56.2 \pm 10.6^{*,+}$ & $51.9 \pm 10.0^{\dagger}$ & $52.1 \pm 11.4^{\dagger}$ \\
$\mathrm{RH}_{\text {upper }}$ & $\%$ & $r=200-800 \mathrm{~km}$ & $p=500-300 \mathrm{hPa}$ & $51.2 \pm 11.1^{*,+}$ & $46.8 \pm 10.6^{\dagger}$ & $46.8 \pm 12.1^{\dagger}$ \\
Vorticity & $10^{7} \mathrm{~s}^{-1}$ & $r=0-1000 \mathrm{~km}$ & $p=850 \mathrm{hPa}$ & $26.5 \pm 54.7^{*,+}$ & $20.4 \pm 59.0^{\dagger,+}$ & $4.6 \pm 58.5^{\dagger, *}$ \\
Divergence & $10^{7} \mathrm{~s}^{-1}$ & $r=0-1000 \mathrm{~km}$ & $p=200 \mathrm{hPa}$ & $36.3 \pm 37.4^{*,+}$ & $28.3 \pm 39.2^{\dagger}$ & $22.1 \pm 38.8^{\dagger}$ \\
\hline
\end{tabular}

LLCPs were identifiable on the downshear side of the storm $54 \%$ of the time. While the upshear and downshear distributions of LLCP locations in Fig. 9a were close in percentage, only $6.8 \%$ of LLCPs covered greater than $50 \%$ of a radial ring. The ring-like appearance in Fig. 9 arose since LLCPs originated in each quadrant, with some propagating around the storm, while others persisted in the same quadrant, including the upshear quadrants. Therefore, compositing all LLCPs together makes it appear as though pulses are axisymmetric in Fig. 9; yet, as Fig. 4 indicates, that is not the case.

Since LLWPs occurred slightly more often in highershear environments, it is not surprising that LLWPs occurred downshear more often $(60.9 \%$ of the time) than LLCPs (Fig. 9b). As for LLCPs, only $6.8 \%$ of LLWPs covered greater than $50 \%$ of a radial ring, and the axisymmetric distribution seen in Fig. $9 b$ is due to their appearing in different quadrants.

\section{e. Differences between LLCP, LLWP, and NP days}

Figures 4-7 show that on days when LLCPs occurred, there is enhanced deep convection in the inner core overnight. To quantify the difference in convection between LLCP, LLWP, and NP days, the mean and one standard deviation of the inner core $(r=0-200 \mathrm{~km})$, overnight (0000-0300 LT) IR brightness temperature values for LLCP, LLWP, and NP days were calculated (Table 1, line 1). A Student's $t$ test was used to determine if statistically significant differences were present at the 99\% confidence level. On days with LLCPs, IR brightness temperature values were, on average, $17^{\circ}$ and $24^{\circ} \mathrm{C}$ colder than on days with LLWPs and NPs, respectively, differences that were found to be statistically significant.

To determine whether the environment on LLCP days was more favorable for overnight deep convection, the mean and one standard deviation of SHIPS variables from 0000 to 0300 LT on LLCP, LLWP, and NP days were calculated (Table 1, lines 2-10) and a Student's $t$ test at the
99\% confidence level was again run to test significance. Overnight, on LLCP days, TCs were over warmer SSTs, had higher $\mathrm{OHC}$, were in more moist environments, had enhanced low-level vorticity, and had enhanced upperlevel divergence. These results indicate that days when LLCPs occurred had more favorable conditions for early morning, inner-core deep convection. The Student's $t$ test indicated that the differences between LLCP and both LLWP and NP days were significant, though they are somewhat subtle. These subtle differences, however, acting across all variables could support enhanced deep convection between 0000 and 0300 LT on LLCP days. On the other hand, only two variables on LLWP days were statistically different from NP days: low-level vorticity and IR brightness temperature. Therefore, it does not appear that the environment was the distinguishing factor for having a LLWP or an NP day.

The differences seen in Table 1 are not due to biases in the time of year, magnitude of shear, or storm intensity. There was no statistically significant difference in the timing of when LLCP, LLWP, or NP days occurred in the calendar year: most LLCP, LLWP, and NP days occurred at the tail end of August, consistent with the peak in Atlantic basin TC activity. There was a statistically significant difference in the intensity experienced on LLCP days compared to LLWP and NP days: LLCP days had storms of higher intensity (Fig. 8a). There was also a statistically significant difference in shear experienced on LLCP days: LLCP days had lower shear (Fig. 8b). When comparing LLCP days to LLWP and NP days of similar intensities and similar shear environments, however, results were quantitatively and qualitatively similar to results shown in Table 1.

\section{Conclusions}

Dunion et al. (2014) found an outward-propagating IR brightness temperature difference cooling feature, which they termed a "diurnal pulse," which was present in the 
major hurricane cases analyzed in their study. This study expanded on their work by including all Atlantic basin TCs over a 36-yr period. An objective metric was created to identify diurnal pulses with similar temporal phasing as Dunion et al. (2014) using GridSat-B1 IR brightness temperature difference fields. Although this objective metric isolated outward propagation, such propagation occurred in unfiltered data as well (appendix A). By creating such a metric, two pulse types were identified: cooling pulses, similar to the pulses found in Dunion et al. (2014), and warming pulses, a previously unidentified pulse of warmer clouds propagating outward following the Dunion et al. (2014) clock.

Overall, pulsing was found to be near ubiquitous, especially in storms of greater than tropical depression intensity. Cooling pulses occurred $71.5 \%$ of the time, with LLCPs occurring $45.1 \%$ of the time and SLCPs occurring $26.4 \%$ of the time. Warming pulses occurred $16.3 \%$ of the time, with LLWPs occurring $8.5 \%$ of the time and SLWPs occurring $7.8 \%$ of the time. This left only $12.2 \%$ with no pulse identified.

The questions outlined in the introduction were answered for both LLCP and LLWP types:

- LLCPs

- LLCP days have an outward-propagating diurnal pulse that moves with a propagation speed between 8 and $14 \mathrm{~m} \mathrm{~s}^{-1}$. These are the pulses that are documented in Dunion et al. (2014).

- On days that reached major hurricane intensity, an LLCP was found $79.5 \%$ of the time. Yet, LLCPs were also present in weaker intensity storms: $64 \%$, $46 \%$, and $24 \%$ of the time on days that reached minor hurricane, tropical storm, and tropical depression intensity, respectively.

- LLCPs occurred slightly more often in low- and moderate-shear environments than in high-shear environments, but there was less of a dependence on shear than intensity.

- Overall, $54 \%$ of LLCPs were detectable on the downshear side of the storm.

- Only $6.8 \%$ of LLCPs covered greater than $50 \%$ of a radial ring. In a composite view, there was a somewhat artificial axisymmetric signature since LLCPs originated in any quadrant and moved around the storm over a given day.

- Enhanced deep convection in the inner core overnight was a key precursor to a cooling pulse. Therefore, it is not surprising that when the environment was more favorable for overnight convection, LLCPs were found.

- LLWPs

- LLWP days also had an outward-propagating diurnal pulse that moved with the same propagation speed of their LLCP counterparts (between 8 and $14 \mathrm{~m} \mathrm{~s}^{-1}$ ).

- LLWPs occurred most frequently in storms that remained tropical depression intensity $(10.1 \%)$ and were least prevalent in storms that reached major hurricane intensity $(2.7 \%)$.

- Again, there was less of a dependence on shear than intensity. Nevertheless, LLWPs occurred most often in high-shear environments.

- The LLWP spatial distribution showed that $60.9 \%$ of LLWPs occurred on the downshear side of the storm.

- As for LLCPs, only $6.8 \%$ of LLWPs covered greater than $50 \%$ of a radial ring. In a composite view, there was a somewhat artificial axisymmetric signature since LLWPs originated in any quadrant and moved around the storm over a given day.

- LLWP and NP days lacked statistically significant differences in their thermodynamic environments.

While this study gives a comprehensive analysis of the frequency and structure of diurnal pulses in the Atlantic basin, there are still many questions that remain unanswered, which this study cannot address. For example, Dunion et al. (2014) found that diurnal pulses are not simply a reflection of the areal expansion of the cirrus canopy, but are often tied to deep convection. The 6-h IR brightness temperature difference differences cannot answer how often a diurnal pulse might be convectively coupled, since it merely shows differences at the top of the cirrus canopy. It is speculated, however, that these pulses, cooling and warming, share a common excitation mechanism, perhaps gravity waves, and differ mainly with respect to whether or not they can be coupled with, or reinforced by, convection. Case studies and numerical modeling experiments are currently under way that will help to answer these, as well as other, questions.

Acknowledgments. The authors thank the two anonymous reviewers for helpful comments about this research. GridSat-B1 IR brightness temperature was acquired from NOAA's National Centers for Environmental Information (https://www.ncdc.noaa.gov/cdr/fundamental/geostationaryir-channel-brightness-temperature-gridsat-b1). All authors were supported by NSF Grant AGS1636799.

\section{APPENDIX A}

\section{Unfiltered 6-h IR Brightness Temperature Signal}

All days were initially examined to determine dominant signals in the unfiltered difference field. Figures A1a-h 
ALL

6-h IR Brightness Temperature Differences
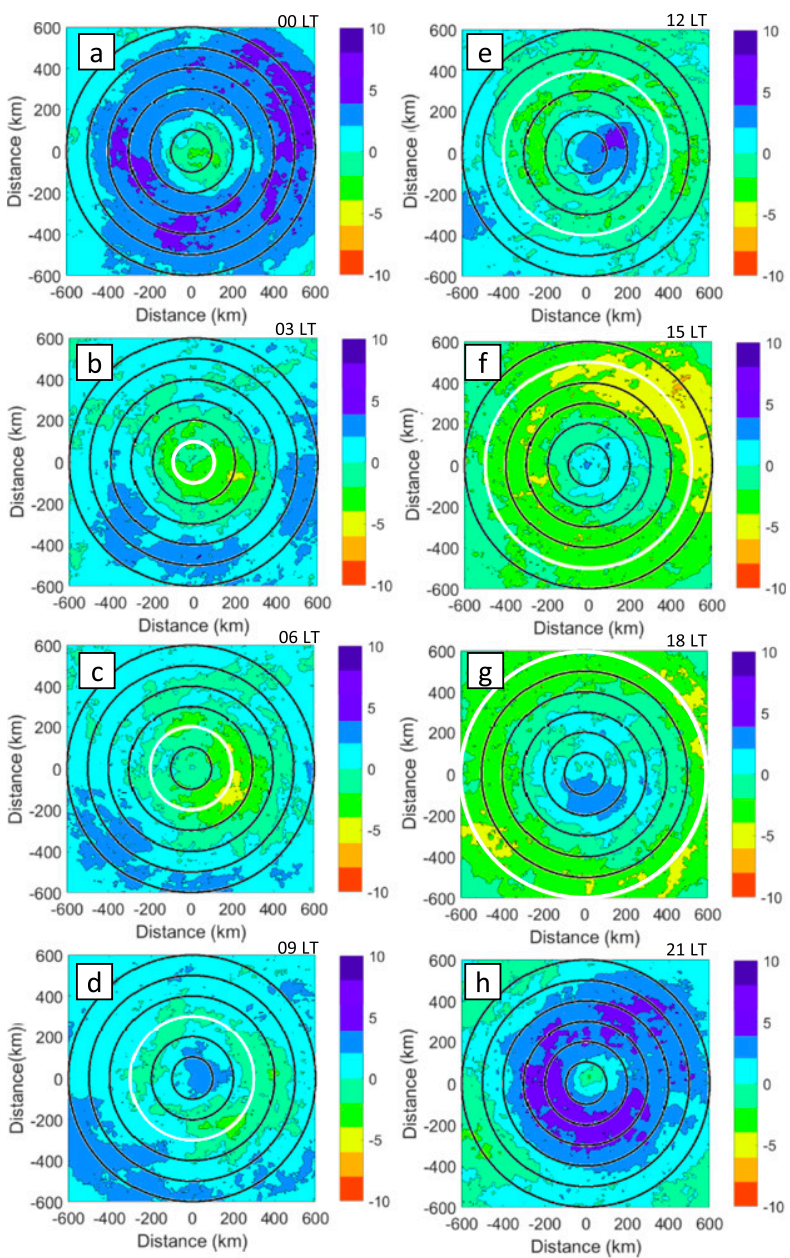

Azimuthal Average
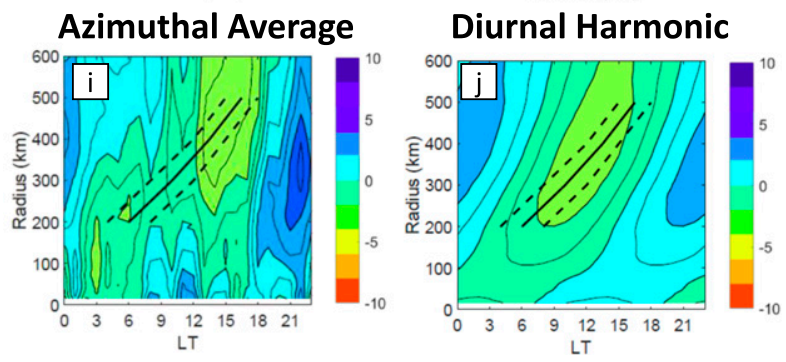

FIG. A1. (a)-(h) As in Fig. 4, but for all days. (i),(j) As in Fig. 7, but for all days.

depict averages of the 6-h IR brightness temperature difference fields for all days for the same eight times shown in earlier figures. An outward-propagating cold pulse appears in the average, even though warm and no pulse days were included. This is most clearly seen in the azimuthal mean Fig. A1i. The corresponding diurnal harmonic (Fig. A1j) is similar in structure to Fig. $7 \mathrm{~b}$, yet weaker since warming pulse and no pulse

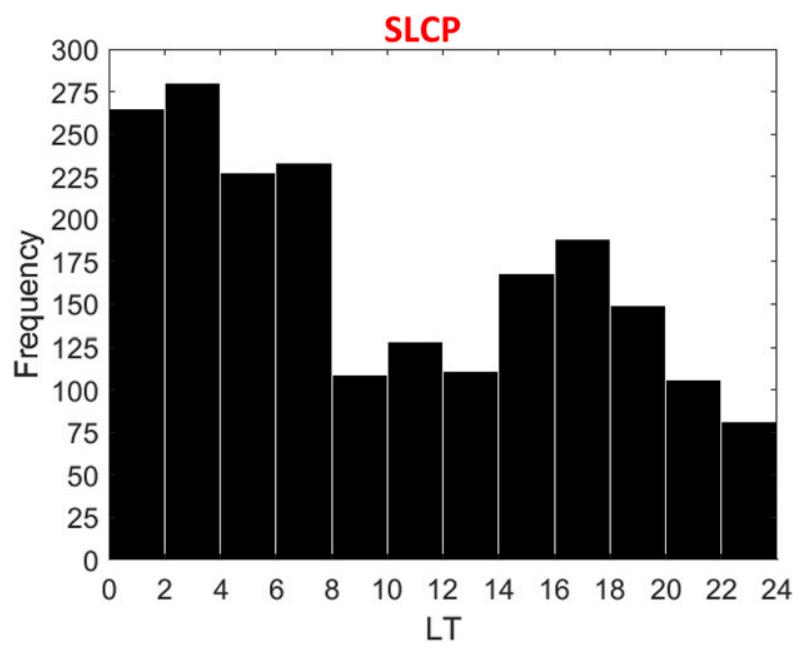

FIG. B1. Histogram (black shading) of the number of times at each LT when SLCPs occurred.

days are included. This evidence suggests that cold pulses are the dominant diurnal signature of tropical cyclones. Thus, creating an objective metric to identify pulses did extract and further highlight the signal.

\section{APPENDIX B}

\section{Short-Lived Pulses}

SLCPs (SLWPs) are defined as days that have cooling (warming) cloud tops that satisfy the areal criterion but only exist for 3-6h. While these pulses do satisfy the IR brightness temperature difference magnitude and areal coverage criteria, and propagate outward, in a composite view their signature is much weaker. Therefore, SLCPs and SLWPs were not the focus of this paper.

The distribution of times where SLCPs occurred is given in Fig. B1. The distribution is bimodal, with one peak occurring between 0200 and 0400 LT and the other peak occurring between 1600 and 1800 LT. Since such pulses are best defined on some days overnight and on other days in the late afternoon, compositing all SLCP days together would show a less defined pulse signature than for LLCP days. This is seen in Fig. B2, which depicts the 6-h IR brightness temperature evolution on days when SLCPs occurred. There is a weak signature of outward-propagating cooling cloud tops over the course of the day. Similar results are found when looking at the distribution of times when SLWPs occurred and the 6-h IR brightness temperature evolution on days where SLWPs occurred (not shown). 


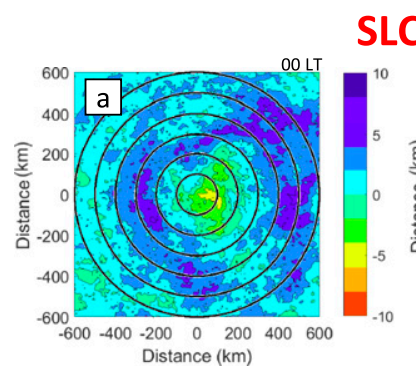

SLCP
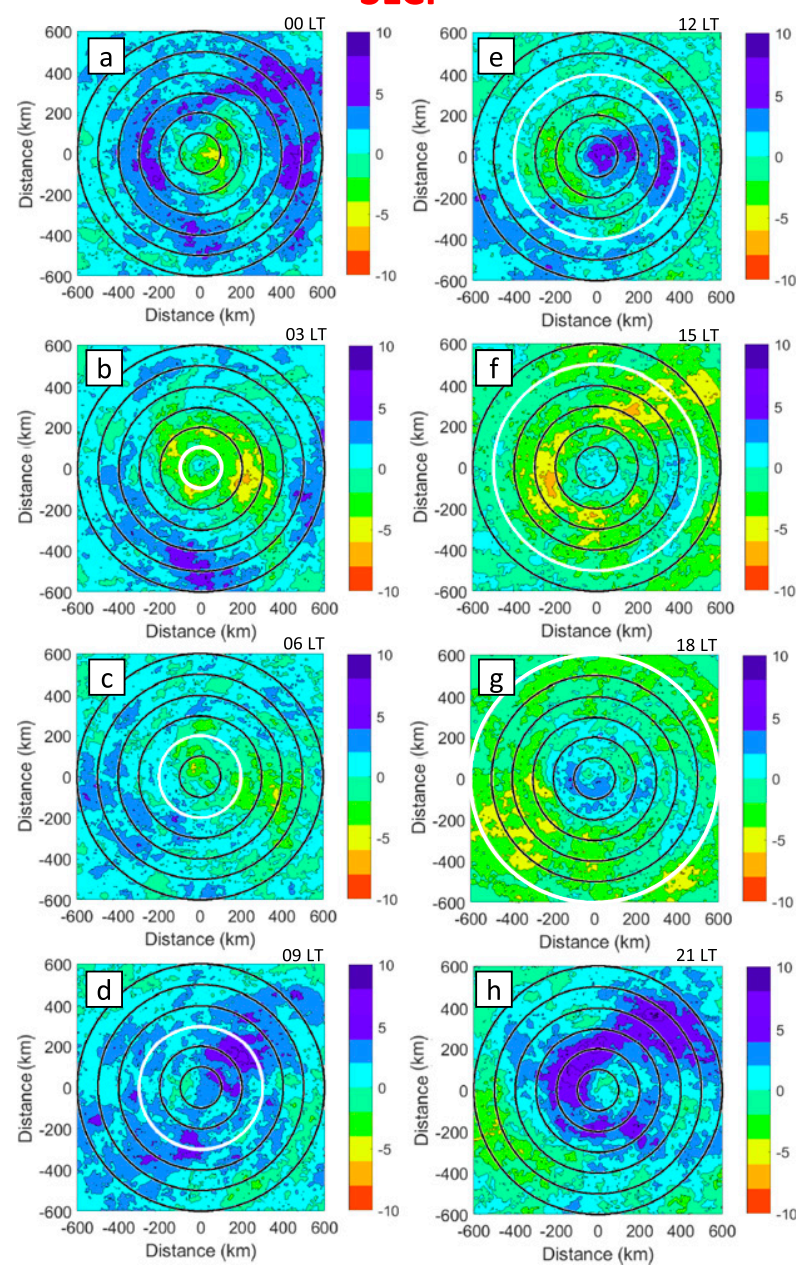

FIG. B2. As in Fig. 4, but for days when SLCPs occurred.

\section{REFERENCES}

Bowman, K. P., and M. D. Fowler, 2015: The diurnal cycle of precipitation in tropical cyclones. J. Climate, 28, 5325-5334, https://doi.org/10.1175/JCLI-D-14-00804.1.

Browner, S. P., W. L. Woodley, and C. G. Griffith, 1977: Diurnal oscillation of the area of cloudiness associated with tropical storms. Mon. Wea. Rev., 105, 856864, https://doi.org/10.1175/1520-0493(1977)105<0856: DOOTAO $>2.0 . \mathrm{CO} ; 2$.

DeMaria, M., and J. Kaplan, 1994: A Statistical Hurricane Intensity Prediction Scheme (SHIPS) for the Atlantic basin. Wea. Forecasting, 9, 209-220, https://doi.org/10.1175/15200434(1994)009<0209:ASHIPS > 2.0.CO;2.

—, and —, 1999: An updated Statistical Hurricane Intensity Prediction Scheme (SHIPS) for the Atlantic and eastern North Pacific basins. Wea. Forecasting, 14, 326-337, https://doi. org/10.1175/1520-0434(1999)014<0326:AUSHIP>2.0.CO;2.

, M. Mainelli, L. K. Shay, J. A. Knaff, and J. Kaplan, 2005: Further improvements to the Statistical Hurricane Intensity Prediction Scheme (SHIPS). Wea. Forecasting, 20, 531-543, https://doi.org/10.1175/WAF862.1.
Dunion, J. P., C. D. Thorncroft, and C. S. Velden, 2014: The tropical cyclone diurnal cycle of mature hurricanes. Mon. Wea. Rev., 142, 3900-3919, https://doi.org/10.1175/ MWR-D-13-00191.1.

Duran, P., and J. Molinari, 2016: Upper-tropospheric low Richardson number in tropical cyclones: Sensitivity to cyclone intensity and the diurnal cycle. J. Atmos. Sci., 73, 545-554, https://doi.org/ 10.1175/JAS-D-15-0118.1.

Frank, W. M., 1977: The structure and energetics of the tropical cyclone I. Storm structure. Mon. Wea. Rev., 105, 11191135, https://doi.org/10.1175/1520-0493(1977)105<1119: TSAEOT $>2.0 . \mathrm{CO} ; 2$.

Ge, X., Y. Ma, S. Zhou, and T. Li, 2014: Impacts of the diurnal cycle of radiation on tropical cyclone intensification and structure. Adv. Atmos. Sci., 31, 1377-1385, https://doi.org/10.1007/s00376014-4060-0.

Hobgood, J. S., 1986: A possible mechanism for the diurnal oscillations of tropical cyclones. J. Atmos. Sci., 43, 29012922, https://doi.org/10.1175/1520-0469(1986)043<2901: APMFTD $>2.0 . \mathrm{CO} ; 2$.

Jiang, H., C. Liu, and E. J. Zipser, 2011: A TRMM-based tropical cyclone cloud and precipitation feature database. J. Appl. Meteor. Climatol., 50, 1255-1274, https://doi.org/10.1175/ 2011JAMC2662.1.

Knapp, K. R., and Coauthors, 2011: Globally gridded satellite observations for climate studies. Bull. Amer. Meteor. Soc., 92 , 893-907, https://doi.org/10.1175/2011BAMS3039.1.

Kossin, J. P., 2002: Daily hurricane variability inferred from GOES infrared imagery. Mon. Wea. Rev., 130, 22602270, https://doi.org/10.1175/1520-0493(2002)130<2260: DHVIFG $>2.0 . \mathrm{CO} ; 2$.

Lajoie, F., and I. Butterworth, 1984: Oscillation of high-level cirrus and heavy precipitation around Australian region tropical cyclones. Mon. Wea. Rev., 112, 535-544, https://doi.org/10.1175/ 1520-0493(1984)112<0535:OOHLCA > 2.0.CO;2.

Landsea, C. W., and J. L. Franklin, 2013: Atlantic hurricane database uncertainty and presentation of a new database format. Mon. Wea. Rev., 141, 3576-3592, https://doi.org/10.1175/MWR-D-1200254.1.

Leppert, K. D., and D. J. Cecil, 2016: Tropical cyclone diurnal cycle as observed by TRMM. Mon. Wea. Rev., 144, 2793-2808, https://doi.org/10.1175/MWR-D-15-0358.1.

Melhauser, C., and F. Zhang, 2014: Diurnal radiation cycle impact on the pregenesis environment of Hurricane Karl (2010). J. Atmos. Sci., 71, 1241-1259, https://doi.org/10.1175/JAS-D13-0116.1.

Muramatsu, T., 1983: Diurnal variations of satellite-measured TBB areal distribution and eye diameter of mature typhoons. J. Meteor. Soc. Japan, 61, 77-90, https://doi.org/ 10.2151/jmsj1965.61.1_77.

Navarro, E. L., and G. J. Hakim, 2016: Idealized numerical modeling of the diurnal cycle of tropical cyclones. J. Atmos. Sci., 73, 4189-4201, https://doi.org/10.1175/JAS-D-15-0349.1.

,-- , and H. E. Willoughby, 2017: Balanced response of an axisymmetric tropical cyclone to periodic diurnal heating. J. Atmos. Sci., 74, 3325-3337, https://doi.org/10.1175/JAS-D16-0279.1.

O’Neill, M. E., D. Perez-Betancourt, and A. A. Wing, 2017: Accessible environments for diurnal-period waves in simulated tropical cyclones. J. Atmos. Sci., 74, 2489-2502, https://doi.org/ 10.1175/JAS-D-16-0294.1.

Shu, H.-L., Q.-H. Zhang, and B. Xu, 2013: Diurnal variation of tropical cyclone rainfall in the western North Pacific in 
2008-2010. Atmos. Oceanic Sci. Lett., 6, 103-108, https:// doi.org/10.1080/16742834.2013.11447064.

Steranka, J., E. B. Rodgers, and R. C. Gentry, 1984: The diurnal variation of Atlantic Ocean tropical cyclone cloud distribution inferred from geostationary satellite infrared measurements. Mon. Wea. Rev., 112, 2338-2344, https://doi.org/10.1175/15200493(1984)112<2338:TDVOAO > 2.0.CO;2.

Stevenson, S. N., K. L. Corbosiero, and S. F. Abarca, 2016: Lightning in eastern North Pacific tropical cyclones: A comparison to the North Atlantic. Mon. Wea. Rev., 144, 225-239, https:// doi.org/10.1175/MWR-D-15-0276.1.

Wu, Q., and Z. Ruan, 2016: Diurnal variations of the areas and temperatures in tropical cyclone clouds. Quart. J. Roy. Meteor. Soc., 142, 2788-2796, https://doi.org/10.1002/qj.2868.

D. Chen, and T. Lian, 2015: Diurnal variations of tropical cyclone precipitation in the inner and outer rainbands. J. Geophys. Res. Atmos., 120, 1-11, https://doi.org/10.1002/ 2014JD022190. 